\title{
Path Choice of Network Social Governance Based on Identity Mapping Relationship
}

\author{
Qi Chen, Dan Wang, Xiangjie Luo \\ Chang'An University, Xinghua College, Shaanxi, Xi’an, 710077, China
}

Keywords: Path choice, Network social governance, Identity mapping relationship

\begin{abstract}
Network technology has been integrated into the texture of social development, affecting the material and ideology of society. Citizen life and national production are readjusted and combined on the structure of network technology, which has spawned a new type of society, namely, a network society. Its virtual, anonymity, openness and decentralized social characteristics weaken the identity of the behavior subject, leading to a series of network anomie behaviors, and at the same time causing the expected effects of governance measures based on subject awareness and identity relationships. By analyzing the actual problems in the governance of the network society, a network identity management path based on the identity mapping relationship is proposed, which is expected to be helpful to the further improvement of China's network society governance.
\end{abstract}

\section{Introduction}

On-line social network OSN (OnlineSocialNetwork) has developed rapidly in recent years. According to StatisticsBrain, Fa-cebok has more than 1 billion registered users and Twiter registered users has reached 5 million; according to statistics, the highest number of concurrent online users in China exceeds 170 million. While OSN brings convenience to people's lives, its anonymity has led to a series of problems such as the flooding of the navy, the spread of rumors, and privacy violations. Such as malicious brush reviews on Taobao, group events caused by rumors, human flesh search events, and so on. In order to solve the anonymity of OSN, some people proposed the network real-name system. The network real-name system refers to a network management system that establishes a one-to-one correspondence between the identity of network users and their personal identities. In essence, it introduces the identity of real society into the network, so that each "network person" can correspond to "real person" stand up. As the first country in the world to fully implement the real-name system, although it was suspended due to the leakage of real-name information, many scholars have studied it. SongDH and others re-evaluated the impact of the regulatory measures taken by the Korean government on the "candlelight march", reflecting the serious obstacles to the freedom of speech of the online real-name system. The analysis of data by ChoD et al. Shows that the real-name system is generally positive for the user's behavior, significantly reducing the user's unconstrained behavior. Analysis by Ohz et al. Shows that real-name system is prone to leak user information and is threatened by phishing websites. Online real-name production is a means of identity authentication, which relies on real-name information to associate "real people" with "network people." In order to fundamentally solve the problem of leakage of real-name information, it is necessary to change the dependency factor required for identity authentication-real-name information cannot be relied on as an authentication factor.

The actors in the network society carry out various connections and activities in the virtual environment, and form relatively stable social connections based on network technology. Together with the real society, they form a parallel social system. The network society has a high degree of generalization that cannot be accommodated by the traditional real society. It not only surpasses the traditional society in form, but the social attributes emphasized by traditional social governance have further shown heterogeneity in the network society, making the network society face unforeseen Behavioral uncertainty and complexity of social relationships. The virtual, anonymity, openness, and decentralized social characteristics of the network society weaken the identity of the 
behavioral subject, leading to a series of network anomie behaviors, and the expectation that governance measures based on subjective consciousness and identity relationship cannot be exerted.

\section{The Necessity of Governance in a Network Society}

Internet Anonymity and Untrusted Network Identity The Internet is designed to be open and anonymous to all users, which provides great convenience for its application and promotion. The anonymity of the Internet is manifested in many ways. First, the communication channel is anonymous, that is, the physical layer is anonymous. The user data is transmitted on the communication channel without any difference during communication, and will not be different due to different individual identities, and no identity information will be added during transmission. Second, the running service is anonymous, that is, the application layer is anonymous. With the development and popularization of the Internet, various websites, forums, chat rooms, etc. provide people with rich network resources. Although a lot of registration is required, the real identity of the user is not checked at the time of registration, and only a user name that is not related to the real identity is used as an identification, so that the network identity displayed by the registered account is anonymous.

Because the network hides people 's true identity, even if they misbehave on the network, they only need to re-register for a new account to get rid of their original identity, which provides conditions for criminals to commit cyber crimes. On the one hand, it lacks a unified identity management mechanism, and on the other hand, the randomness of network use greatly reduces the credibility of the identity, which has caused many security risks, such as identity fraud, identity theft, cyber fraud, cyber slander, cyber rumors, and social panic. Many events such as manufacturing occur frequently. The root cause of these problems is that there is no connection between the individual's network identity and the individual's true identity. The network identity is just a string and does not represent any real component. Therefore, users do not need to be responsible for their own network behavior, which increases the wantonness and uncontrollability of network behavior, and even triggers malicious and criminal behavior.

The Internet's requirements for trusted identities continue to rise. Although the use of false identities on the Internet has implicitly become a consensus reached by people, at the same time, the occurrence of various network security incidents has also caused people to worry about the use of real identities on the Internet. However, with the continuous promotion of networkization, the negative impact of anonymity has become increasingly prominent, and the demand for trusted network identities has been increasing. Although anonymity has played a role in the development of the network, user interactions in virtual communities such as online forums and chat rooms have also exposed the shortcomings of online communication, specifically: Social situations in which social norms are lacking, ambiguous, or changeable and cannot provide guidance to members of society. "In the real world, the supervision of acquaintances, the pressure of public opinion, and the deterrence of legal sanctions have formed a strong binding force and formulated intangible rules for interpersonal communication. With the loss of restraint in the online world, individual behaviors gradually break away from all kinds of restraints and become uncontrolled, and even develop into malicious or criminal acts.

Online social networks have promoted the development of trusted network identities. Online social networks have involved work, entertainment, leisure and other aspects, making the network closer to people's lives and inseparable. This not only changes the way people use the Internet, but also speeds up the process of personalizing information online. People tend to expose more personal information on the Internet in exchange for more personalized services, and this personal information is concentrated in the identity of the network. In face-to-face communication, selfdisclosure helps to create a sense of trust, which in turn can increase the courage of self-disclosure, thus forming a virtuous circle and closer relations between the two parties. Self-disclosure of real identity is an important means to reduce cognitive uncertainty in interpersonal communication on the Internet, and is the basis for establishing a trust mechanism. The survey found that $60.5 \%$ of the respondents believed that understanding the true identity and social status of netizens would 
increase mutual trust and thus communicate more frankly.

\section{The Impact of the Failure of Identity Mapping on the Governance of Network Society}

China's national informatization development strategy has made a comprehensive deployment for the construction of an information security guarantee system, and proposed strategic tasks at the technical, management, and legal levels. Network real identity management has become an urgent problem to be solved. In the face of increasingly severe cybersecurity threats, scholars have tried to explain the root causes of network anomie behaviors from various levels, such as moral loss, legal absence, credibility crises, and weak management. In order to regain the scepter of order. It is true that these disadvantages are the inducement of network anomie behavior, and the continuous improvement of various levels will undoubtedly contribute to the healthy development of the network society. However, the author believes that various social issues in the governance of network society can be brought together into a focus, which is also the most fundamental crux of network anomie behavior, that is, the identity mapping is invalid.

The identity of the actual social behavior subject is unique. Whether it is moral guidance or legal regulation, it has a specific role, which can achieve the effect of restraint or deterrence. In the network society, due to "behavior digitization" and "subject virtualization", the real social identity of the actors and the network social identity form a 1: $\mathrm{N}$ mapping relationship. At the same time, the identity relationship of the network society also overlaps. Identity mapping is further complicated into $\mathrm{N}$ : $\mathrm{N}$ mapping relationships, which can easily cause the behavioral consciousness of network social subjects to deviate from the track of order and produce various network anomie behaviors. Generally, scholars tend to use the theory of network ethics to describe this fact. Although "ethics and morality is a category combining universality and particularity", the identity mapping relationship contains much more content than "ethics". "Ethics" often makes value judgments based on various subjective factors, emphasizing the application of subjective initiative in the rules of behavior. However, it is undeniable that the anomalous behavior of the network often also depends on the objective environment. The influence of external factors on the subject must also be considered. Identity mapping can be better subjective and objective, and it is more targeted and universal for problem solving. Sex. Identity mapping is, in fact, a reconstruction of a correspondence relationship, which aims to weaken the uncertain impact of network virtuality on subject behaviors by constructing an identity management system corresponding to that in the real world in a network society, and restricting anomie behavior to morality and law To achieve consistency in the awareness, behavior, and responsibility of the subject of the network society. The way of governance in the real society is to apply the effects of moral and legal constraints on specific subjects, to achieve the control and adjustment of subject behaviors with a negative evaluation of subject behaviors. The basis for their effectiveness is the determination of the identity relationship corresponding to the subjects. Sex. Simply put, the behavior of real society can always be traced back to the only and specific subject, and the constraint mechanisms such as morality and law can produce deterrent and normative effects. In the network society, due to the fictitious nature of the identity of the subject, the correspondence between the above actions and the subject is broken, and the normative effects of morality and law cannot be directed to the identified subject, so that governance measures often face an embarrassing situation that cannot be applied. The ineffectiveness of online social identity mapping will have a diffuse negative impact, which will have an impact on the traditional social norm system that cannot be ignored.

\section{The Path of Network Social Governance Based on Identity Mapping}

Reconstructing the identity mapping relationship between the actual society and the actors of the network society is the most effective path to solve the governance problems of the network society. Virtual identity (online identity) is an extension of the real identity of a natural person in the virtual world. The essence of identity mapping relationship is the extended relationship between identity and identity. In real society, the identity information of natural persons can be verified and 
confirmed through valid documents, etc., but in the network environment, due to the lack of identity proof facts, the virtual nature of identity and behavior weakens the regulation of the network society, thus making the network moral The huge impact of law, integrity and integrity is the root cause of the aforementioned negative impacts and risk challenges. Identity mapping refers to a system that establishes a correspondence relationship between the identity of a social subject on the Internet and the real identity of an individual in the real world through electronic identity identification, digital signatures, and data encryption techniques based on compulsory identity authentication. By establishing this identity correspondence, the moral self-restraint mechanism can be restored, and the role of laws in regulating and guiding behavior can also be exerted.

There are many ways to achieve this kind of identity mapping relationship, and the network realname system is one of the solutions that has received widespread attention. The real-name system of the Internet provides effective identification through the subject as a prerequisite for network activities. From Internet cafes' real-name system, to college BBS real-name system, to Weibo realname system, the scope of application of real-name system is constantly expanding, which has also triggered the standardization of online order. In view of the public's deep concerns about freedom of speech and privacy protection, people's trust in the online society is far from being able to transparentize their information. Therefore, if the online real-name system is enforced, it will inevitably infringe on the private rights of the public, lose existing or potential Internet users, and cause a personal information protection crisis because public information is too transparent. Therefore, while implementing the online real-name system, it is necessary to take corresponding legal and technical guarantee systems as the basis to prevent possible risks. For example, South Korea is the first country in the world to implement the real-name system of the Internet, and has achieved good results in the governance of the Internet society. By extending the normative system of the real society to the Internet society, it has achieved the goal of regulating network behavior and purifying cyberspace. However, before implementing the online real-name system, South Korea first formulated or modified supporting laws and regulations, such as the Basic Law for the Promotion of Informatization, the Basic Information and Communications Protection Law, and the Telecommunications Business Law, to ensure the protection of public rights and interests after the implementation of the real-name system. Will be impacted. At the same time, in order to prevent the appearance of false identity information, a complete set of identity authentication systems has been established to ensure the authenticity and accuracy of user identity information. At present, China does not have corresponding guarantee measures in terms of legal protection system and technical certification system, and there is still a long way to go to realize the real-name system on the Internet.

\section{Conclusion}

The core of realizing the identity mapping relationship is to establish a correspondence relationship, as long as the identity of the network social subject and the identity information in the real society can be unified and its authenticity can be achieved. Therefore, we can bypass the "anonymous-real name" logic and adopt a more direct confirmation system. The identity management of the actual social subject is more intuitive and convenient, because the identity information of each person is unique and specific, just as the IP address of each computer is unique, so it can be traced back to the computer through the IP address. Then, as long as the uniqueness and specificity of the identity information of the network social subject are realized, the dilemma of invalidation of identity mapping is also solved.

\section{Acknowledgment}

2019 Special Scientific Research Program of Shaanxi Provincial Department of Education Groject name:'Exploration of Basic Thoughts and Safeguarding Norms of Dual Construction of Morality and Law in Network Society'Groject number: 19JK1016 


\section{References}

[1] Li Fang. Network Communication and Reconstruction of Audience's Personality [J]. Journal of Shenyang Institute of Education, 2004, 01: 68-70.

[2] Bo Mei. On the Psychological Compensation Function of Network Culture [J]. Exploration of Psychology, 2004,04: 17-19.

[3] Wang Xiaoxia. Interpersonal Communication in "Virtual Society" and Its Adjustment [J]. Journal of Nankai University, 2002, 04: 88-94.

[4] DONATHJ, BOYDD.Publicdisplaysofconnection [J] .bttechnology Journal, 2004, 22 (4): 71-82.

[5] Shi Lei. The influence of personal real identity in interpersonal communication on the Internet [J]. Contemporary Communication, 2007, 01: 57-9. 